https://helda.helsinki.fi

\title{
Knee dislocation in overweight patients
}

\section{Peltola, Erno}

\section{9}

Peltola , E , Lindahl , J , Hietaranta , H \& Koskinen , S K 2009 , ' Knee dislocation in overweight patients ' , American Journal of Roentgenology , vol. 192 , no. 1 , pp. 101-106 . https://doi.org/10.2214/A

http://hdl.handle.net/10138/279385

https://doi.org/10.2214/AJR.07.3593

publishedVersion

Downloaded from Helda, University of Helsinki institutional repository.

This is an electronic reprint of the original article.

This reprint may differ from the original in pagination and typographic detail.

Please cite the original version. 


\section{Knee Dislocation in Overweight Patients}

\section{Erno K. Peltola ${ }^{1}$ Jan Lindahl ${ }^{2}$ Harri Hietaranta ${ }^{3}$ Seppo K. Koskinen ${ }^{1}$}

Keywords: injuries, knee dislocation, MRI, obesity

DOI:10.2214/AJR.07.3593

Received December 27, 2007; accepted after revision June 23, 2008 .

'Department of Radiology, Helsinki Medical Imaging Center, Helsinki University Hospital, Töölö Trauma Center, Topeliuksenkatu 5, Helsinki, PL 266, 00029 HUS, Finland. Address correspondence to E. K. Peltola (erno. peltola@fimnet.fi).

${ }^{2}$ Department of Orthopaedics and Traumatology, Helsinki University Hospital, Töölö Trauma Center, Helsinki, Finland.

${ }^{3}$ Klinikka 22 oy, Helsinki, Finland.

AJR2009; 192:101-106

0361-803X/09/1921-101

(c) American Roentgen Ray Society

OBJECTIVE. The purpose of this study was to evaluate the incidence, cause, injury patterns, and MRI findings in knee dislocation in patients with normal and increased body mass index and to determine whether obesity interferes with knee MRI examinations.

MATERIALS AND METHODS. A retrospective study of the period from 2000 to 2007 (90 months) was performed at a level 1 trauma center, finding a total of 24 patients who had sustained a knee dislocation.

RESULTS. Twenty-two of the 24 patients underwent surgery and 19 patients had an MRI examination of diagnostic quality before surgery. Of the 24 patients, 11 had a body mass index greater than 25 and had knee dislocation due to low-energy trauma (nine due to a simple fall, two to a noncontact sport). Two of these 11 patients were morbidly obese (body mass index > 40). These patients had no injuries to the popliteal tendon and they had no irreversible peroneal nerve injuries. Otherwise, the patients' injuries were in agreement with previous knee dislocation studies. Obesity did not interfere with knee MRI examinations. On the basis of the population served by our trauma center, the annual incidence of knee dislocation due to low-energy trauma in overweight patients is about 1.0 per million.

CONCLUSION. The annual incidence in obese patients of knee dislocation due to lowenergy trauma is not insignificant at a level 1 trauma center. As the prevalence of obesity increases, the injury patterns seen in emergency departments may change. The radiologist should be aware that even after a simple fall, overweight patients may have a knee dislocation.

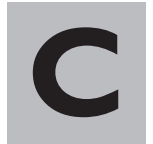

omplete dislocation of the knee is an uncommon trauma $[1,2]$. Because of the potentially severe neurovascular damage, knee dislocation can be limb-threatening [2-4], and it is important to make the correct diagnosis without delay. Often conventional radiography is not diagnostic because of spontaneous reduction, making the diagnosis difficult [5-7]. MRI provides good assessment of the extent of soft-tissue injury $[6,8,9]$, and the radiologist has an important role in recognizing injury patterns of knee dislocation on MR images.

Dislocation of the knee usually results from high-energy trauma such as motorcycle and motor vehicle accidents or a sports-related injury $[1,2,4,6,8,10]$. However, if the knee dislocation is due to an unusual cause, such as a simple fall in an obese patient [11, 12], the correct diagnosis may be more difficult to make. As the prevalence of obesity increases in Western Europe and in the United States [13-15], the frequency of obesityrelated injuries may increase.
The purpose of this study was to evaluate the incidence and cause of knee dislocation in patients with normal and increased body mass index (BMI), to assess MRI findings in knee dislocation, to determine whether patients with increased BMI have any specific injury patterns, and to determine whether the presence of obesity interferes with knee MRI examinations.

\section{Materials and Methods}

This retrospective study was performed at a level 1 trauma center and was approved by the institutional review board. Patients with knee dislocations from April 2000 to the end of September 2007 were identified using the hospital's PACS and the hospital discharge register. Using PACS, we retrieved all of the hospital's requests for knee MRI studies and looked for the term "knee dislocation" in the report. In our hospital, early preoperative MRI is routinely performed on patients with knee dislocation, and all MRI requests include a brief history and clinical indication stated by the referring physician. If the 
examination is interrupted (e.g., due to claustrophobia), the MRI request still remains in the PACS. In addition, we searched the hospital discharge register for the diagnosis of knee dislocation (ICD-10 [International Classification of Disease] code S83.1).

An approximation of the annual incidence of knee dislocation in adults was calculated by comparing identified patients having knee dislocation with the general population of 1.4 million people. Our hospital is the only level 1 trauma center serving this population; no patients younger than 16 years are treated at our hospital.

The MR images were obtained with a 1.5-T unit (Signa MRI EchoSpeed, GE Healthcare). Two different dedicated knee coils were used: the quadrature lower extremity coil (Medical Advances; internal diameter, $18 \mathrm{~cm}$ ) and an 8-channel HD knee array coil (MRI Devices Corporation; internal diameter, $19 \mathrm{~cm}$ ).

The standard sequences used were coronal T2weighted fast spin-echo with fat saturation, sagittal proton-density spin-echo, sagittal T2-weighted

TABLE I: Age, Sex, Body Mass Index (BMI), and Cause of Knee Dislocation in 24 Patients

\begin{tabular}{|c|c|c|c|c|}
\hline Patient No. & Age (y) & Sex & BMI & Cause of Injury \\
\hline 1 & 61 & M & 36 & Fall \\
\hline 2 & 39 & $\mathrm{~F}$ & 30 & Downhill skiing \\
\hline 3 & 33 & M & 27 & Fall \\
\hline 4 & 64 & M & 32 & Fall \\
\hline 5 & 33 & $\mathrm{~F}$ & 22 & Horseback riding \\
\hline 6 & 53 & $\mathrm{~F}$ & 33 & Fall \\
\hline 7 & 57 & M & 33 & Downhill skiing \\
\hline 8 & 29 & M & 24 & Fall \\
\hline 9 & 43 & M & 22 & Downhill skiing \\
\hline 10 & 64 & $\mathrm{~F}$ & 29 & Fall \\
\hline 11 & 25 & M & 19 & Running while carrying an adult \\
\hline 12 & 53 & $\mathrm{~F}$ & 23 & Motor vehicle accident \\
\hline 13 & 35 & $\mathrm{~F}$ & 20 & Jumping, sport-related \\
\hline 14 & 62 & M & 19 & Bicycling \\
\hline 15 & 38 & M & 20 & Motor vehicle accident \\
\hline 16 & 41 & $\mathrm{~F}$ & 42 & Fall \\
\hline 17 & 59 & M & 29 & Fall \\
\hline 18 & 16 & M & 23 & Motor bike accident \\
\hline 19 & 27 & $\mathrm{~F}$ & 42 & Fall \\
\hline 20 & 25 & $\mathrm{~F}$ & 28 & Motor vehicle accident \\
\hline 21 & 59 & $\mathrm{~F}$ & 23 & Motor vehicle accident \\
\hline 22 & 23 & M & 22 & Motor vehicle accident \\
\hline 23 & 46 & M & 36 & Fall \\
\hline 24 & 39 & M & 30 & Motor bike accident \\
\hline
\end{tabular}

fast spin-echo, and axial proton-density fast spinecho with fat saturation sequences. The fatsuppressed T2-weighted fast spin-echo sequence was performed with TR/effective TE of 4,700/40, a 16 -cm field of view, a $512 \times 512$ matrix, a $4-\mathrm{mm}$ slice thickness, 3 excitations, and an echo-train length of 8 . The $\mathrm{T} 2$-weighted fast spin-echo sequence was performed with 4,000/88, $14-\mathrm{cm}$ field of view, $512 \times 512$ matrix, 3-mm slice thickness, 2 excitations, and an echo-train length of 16. The proton-density spin-echo sequence used 1,800/20, 14-cm field of view, $256 \times 256$ matrix, 3-mm slice thickness, and 1 excitation. The fat-suppressed proton-density fast spin-echo sequence used 3,000/26, 14 -cm field of view, $512 \times$ 512 matrix, 3-mm slice thickness, 3 excitations, and an echo-train length of 6 . Because the orthopedic surgeons at our institution prefer conventional angiography over MR angiography, conventional angiography was performed when signs of vascular injury were present.

Two radiologists, one with less than 1 year of experience in musculoskeletal radiology and the other with more than 10 years' experience in musculoskeletal MRI, independently reviewed the MR images. Equivocal cases were decided by consensus.

Subjective image quality was independently assessed by two readers. Images were classified as either diagnostic or nondiagnostic. In our hospital, subjective image quality is routinely evaluated by a radiologist. If image quality is nondiagnostic, it is mentioned in the radiologist's report. Therefore, for evaluation of subjective image quality, we also reviewed the MRI reports of those patients who were included in the current study.

The MRI studies were evaluated for the presence of injury to the anterior cruciate ligament (ACL), posterior cruciate ligament (PCL), medial collateral ligament (MCL), lateral collateral ligament (LCL), popliteus tendon, biceps femoris tendon, quadriceps tendon, patellar ligament, and medial retinaculum. If present, injury was categorized as partial or complete. Associated injuries to the bone, menisci, cartilage, peroneal nerve, and popliteal artery were also analyzed. MRI findings were compared with surgical findings.

BMI and mechanism of injury were retrieved from each patient's file. The annual incidence of knee dislocations, the spectrum of injuries, and the sensitivity and specificity of MRI to detect injuries of overweight $(\mathrm{BMI}>25)$ patients with knee dislocation due to low-energy trauma were examined.

The differences in injuries between the two patient groups (those with BMI $>25$ and those with normal BMI) were analyzed using the Mann-Whitney $U$ test (nonparametric data). A $p$ value of less than 0.05 was interpreted as statistically significant. The SAS/STAT 8.02 (SAS Institute) program software was used for the statistical computations.

\section{Results}

Incidence and Cause of Knee Dislocation

During the 90-month period, a total of 24 patients (14 male and 10 female; age range, 16-64 years; mean age, 42 years) who had sustained a knee dislocation were identified (Table 1). Using the assumptions described in Materials and Methods, we estimated the annual incidence of knee dislocations in adults at 2.3 per million. Patients were included in the study if they had dislocation on radiographs $(n=17)$ or if they had a well-documented description of the dislocation in their patient files $(n=7)$. Patients younger than 16 years were not included because they are not treated at our hospital. Twenty-two patients underwent surgery. One patient died before the knee surgery, 
Knee Dislocation in Overweight Patients

TABLE 2: Cause of Injury and Body Mass Index in 24 Patients with Knee Dislocation

\begin{tabular}{l|c|c|c|c}
\hline \multirow{2}{*}{\multicolumn{1}{c}{ Cause of Injury }} & \multicolumn{4}{|c}{ Patient Body Mass Index } \\
\cline { 2 - 5 } \multicolumn{1}{c}{} & $\begin{array}{c}\text { Normal } \\
(18.5-24.9)\end{array}$ & $\begin{array}{c}\text { Overweight } \\
(25-29.9)\end{array}$ & $\begin{array}{c}\text { Obese } \\
(30-39.9)\end{array}$ & $\begin{array}{c}\text { Morbidly Obese } \\
(>40)\end{array}$ \\
\hline Motor vehicle or motorcycle accident & 5 & 2 & & \\
Sports-related & 5 & 1 & 4 & 2 \\
Simple fall & 1 & 3 & 4 & 2 \\
\hline
\end{tabular}

Note-Data are numbers of patients.

and one patient's knee was not operated on because of extensive muscle necrosis due to vascular injury and compartment syndrome. Nineteen patients had an MRI examination of diagnostic quality before surgery (mean, 5 days; range, 0-26 days). Patient mean BMI was 28 (range, 19-42).

Of the 24 patients, seven had knee dislocation caused by high-energy trauma such as a motor vehicle or motorcycle accident. Two of these high-energy trauma patients were overweight (BMI, 25-29.9) and five had a normal weight (BMI, 18.5-24.9). Seven patients had knee dislocation due to athletic injury. Five of these patients had normal weight, one was overweight, and one patient was obese (BMI, 30-39.9). Ten patients had dislocation due to a simple fall. Of these patients, one had normal weight, three were overweight, four were obese, and two were morbidly obese (BMI > 40) (Table 2).

A BMI of less than 25 is considered normal [16]. Eleven patients (five women, six men; age range, 27-64 years; mean age, 49 years) had a BMI greater than 25 (mean, 33; range, 27-42) and had knee dislocation due to low-energy trauma (nine due to a simple fall, two due to downhill skiing). These patients were of special interest in this study and are referred to as group A. The other 13 patients - patients with dislocation due to high-energy trauma $(n=7)$ and normal-weight patients with dislocation due to low-energy trauma $(n=6)$ - are referred to as group B (mean BMI, 22; BMI range, 19-28; five female; eight male; age range, $16-62$ years; mean age, 36 years). The annual incidence of patients having a BMI greater than 25 and had knee dislocation due to low-energy trauma is about 1.0 per million.

\section{MRI Findings, Operative Findings, and Image Quality}

The subjective quality of all MRI studies was classified as diagnostic by both readers. In the reviewed original reports, there was no mention of nondiagnostic image quality. In every reviewed report, a radiologist was able to answer the questions that the clinician had asked in the request.

Operative findings revealed that all 22 patients had a complete tear of the ACL. All patients in group A had also a complete tear of the PCL. In group B, one patient had an intact PCL and all others had a complete tear of the PCL. Complete tear of the MCL was present in seven and five patients in groups A and B, respectively. A complete tear of the LCL was found in five patients in both groups.

Complete popliteal tendon tears occurred in five patients, all of whom were in group B $(p=0.0262)$. One patient from group B had a complete rupture of the biceps femoris tendon. There was no complete tear of the quadriceps femoris tendon or patellar tendon in any patient. Complete rupture of the medial retinaculum was identified on MRI examinations in 10 patients.

MRI evaluation of the ligaments and tendons resulted in one false-negative and one false-positive diagnosis of a complete tear in group A (both LCLs). In group B, there were one false-negative (ACL) and two false-positive (one MCL and one LCL) diagnoses of complete tears. Operative findings are shown in Table 3.

Bone, cartilage, and meniscal injuriesTen avulsion fractures and one tibial plateau fracture were seen in eight patients. Two of these fractures were in group A and nine in group B. Six avulsion fractures of the intercondylar eminence of the tibia (four PCL and two ACL) and four avulsion fractures of the proximal fibula (all LCL) were seen. One patient's lateral tibial plateau was fractured. MRI evaluation of the fractures resulted in one false-positive diagnosis of avulsion fracture of the tibia.

Chondral injuries were classified into four grades according to the evaluation system of the International Cartilage Repair Society [17]. Nine grade I, two grade II, and one grade III injuries were observed at surgery. Of these 12 chondral injuries, eight were found in patients in group A. MR images were evaluated for the presence of an osteochondral fracture or a chondral loose fragment, but none was found.

Patients in group A had eight meniscal tears; group B patients had four meniscal tears. Of the meniscal tears, four were medial and eight were lateral. Three meniscal tears were radial, one was horizontal, and four were vertical. One bucket-handle tear was seen, and three menisci had ruptured with a displaced fragment. MRI evaluation resulted in four false-positive diagnoses of meniscal tears (one vertical, two radial, and one with a displaced fragment). False-positive MRI diagnoses were equally divided between the two groups (Fig. 1).

No statistically significant differences in bone, cartilage, or meniscal injuries between the two groups were found.

Neurovascular injuries-Three patients had signs of complete peroneal nerve injury after knee dislocation. Early nerve reconstruction surgery was performed in one patient. None of these three patients has regained peroneal function after 1 year of follow-up. All three patients belonged to group B.

Four patients had signs of partial peroneal injury: weak ankle dorsiflexion in three and paresthesias in one. After 1 year of followup, three of these patients regained function with rehabilitation. Two patients were in group A and two in group B.

\section{TABLE 3: Surgical Findings in 22 Patients with Dislocated Knees}

\begin{tabular}{l|c|c}
\hline \multicolumn{1}{c|}{ Finding } & $\begin{array}{c}\text { Group A } \\
(n=10)\end{array}$ & $\begin{array}{c}\text { Group B } \\
(n=12)\end{array}$ \\
\hline ACL rupture & 10 & 12 \\
PCL rupture & 10 & 11 \\
MCL rupture & 7 & 5 \\
LCL rupture & 5 & 5 \\
Popliteal tendon rupture & 0 & 5 \\
Biceps femoris tendon & 0 & 1 \\
rupture & & \\
Medial meniscal tear & 3 & 1 \\
Lateral meniscal tear & 5 & 3 \\
Chondral injury & 8 & 4 \\
Fracture & 2 & 9 \\
\hline
\end{tabular}

Note-Group A, patients with body mass index $>25$ and dislocation due to low-energy trauma; group $B$, patients with high-energy trauma or normal-weight patients with low-energy trauma. $\mathrm{ACL}=$ anterior cruciate ligament, $\mathrm{PCL}=$ posterior cruciate ligament, $\mathrm{MCL}=$ medial collateral ligament, $\mathrm{LCL}=$ lateral collateral ligament.

${ }^{\text {a }} p=0.0262$ 
Peltola et al.

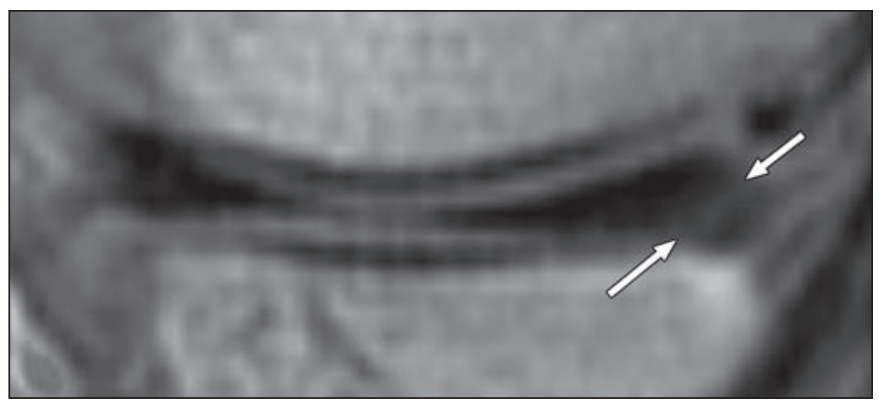

A

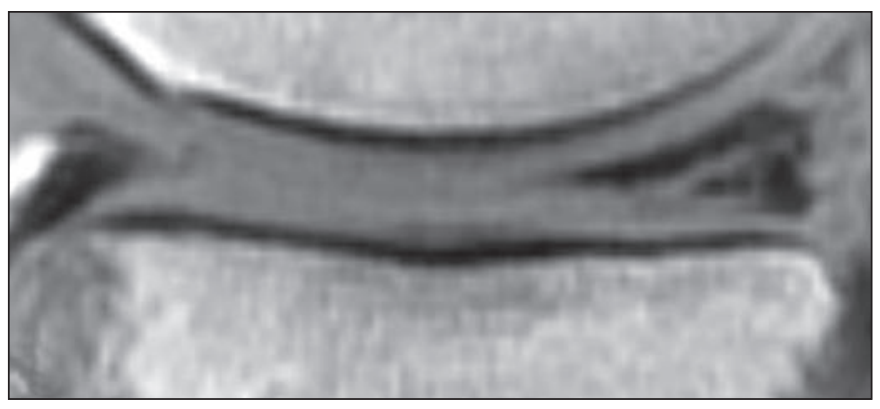

B

Fig. 1-Two patients with knee injuries.

A, In 53-year-old woman (patient 12), faint line (between arrows) seen in posterior horn of medial meniscus was interpreted as tear. At surgery, meniscus was intact.

B, In 64-year-old woman (patient 10), sagittal image through lateral meniscus reveals true-positive oblique horizontal tear of posterior horn.

On MR images, seven patients had edema or hematoma surrounding the peroneal nerve. Two of these patients had clinical signs of a partial peroneal injury. On the MRI examinations of seven patients, the peroneal nerve was interpreted as normal; none of these patients were symptomatic from nerve injury. Of the three patients with complete irreversible peroneal paresis, only one had an MRI examination before surgery. In this patient, the peroneal nerve was not identified on the MR images.

Two patients in group A and one patient in group B had popliteal artery injuries. In these

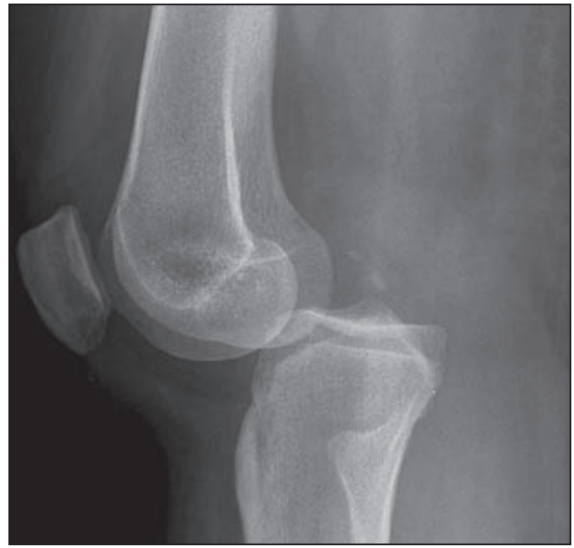

A

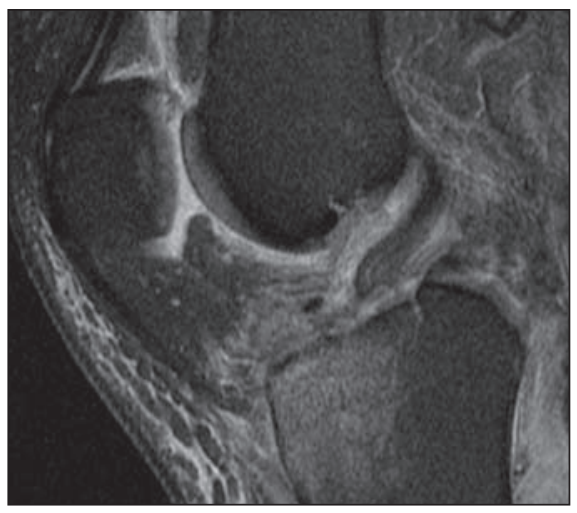

B patients, vascular injury was suspected at the time of admission, and they underwent subsequent conventional angiography and were treated surgically. All three patients had return of blood flow after surgery, and no reoperations or amputations were necessary. None of the patients underwent MR angiography.

\section{Discussion}

Incidence and Cause of Knee Dislocation in Patients with Normal and Those with Increased BMI

In the literature, dislocation of the knee has been reported to occur mainly as a result of high-energy trauma or contact-sport-related trauma $[1,2,4,6,8,10]$. Single case reports of low-energy knee dislocation in obese patients have been published $[11,12]$. In our study, however, $46 \%$ of patients with knee dislocation were overweight and suffered low-energy trauma. One reason for this difference is the strict inclusion criteria of our study. We included only patients with dislocation on radiographs or patients with clearly documented dislocation. For example, patients with a dislocated knee at surgery but who had no evidence of previous true dislocation were not included. Most likely, some spontaneously reduced dislocations were not included in this study because there was no documented evidence of dislocation or because the patient could not remember the knee dislocation because of a high-energy multitrauma. Other possible explanations for the difference are the increasing prevalence of obesity [13-15] and the evolution of safety mechanisms in motor vehicles. Nevertheless, our study shows that the annual incidence of knee dislocation due to low-energy trauma in obese patients is not insignificant at a level 1 trauma center (Fig. 2).

A downhill skiing accident can be either low- or high-energy trauma. In our study, all three downhill skiing trauma patients were

Fig. 2-27-year-old woman who slipped while walking and had complete dislocation of knee. Patient's body mass index was 42.

A, Conventional radiograph shows posterior dislocation of knee.

B-D, MR images show complete rupture of anterior and posterior cruciate ligaments and grade II rupture of medial collateral ligament (arrowhead, D). Findings were verified surgically. Note good diagnostic quality of images despite patient's obesity.
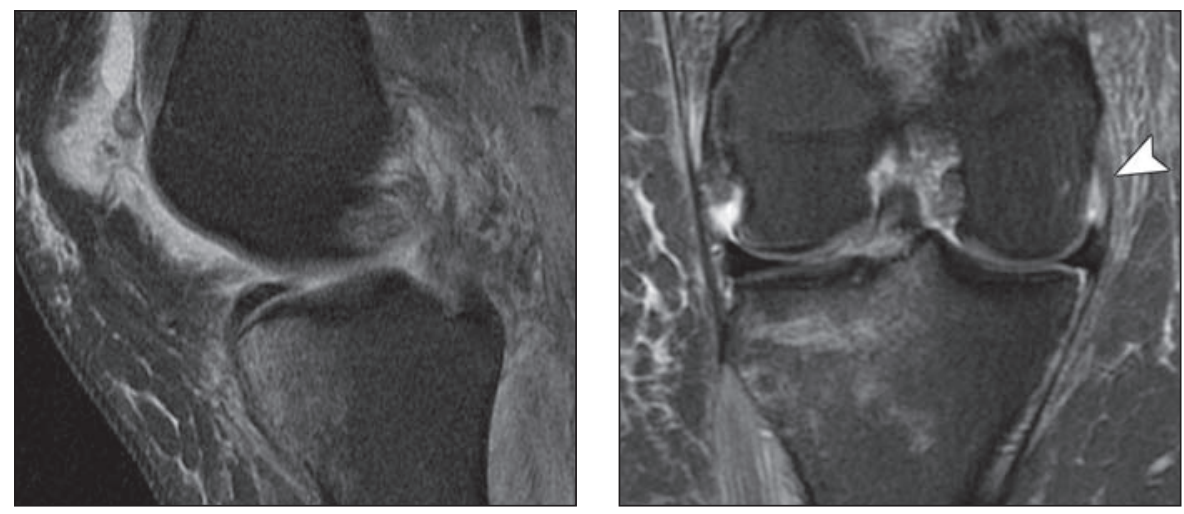


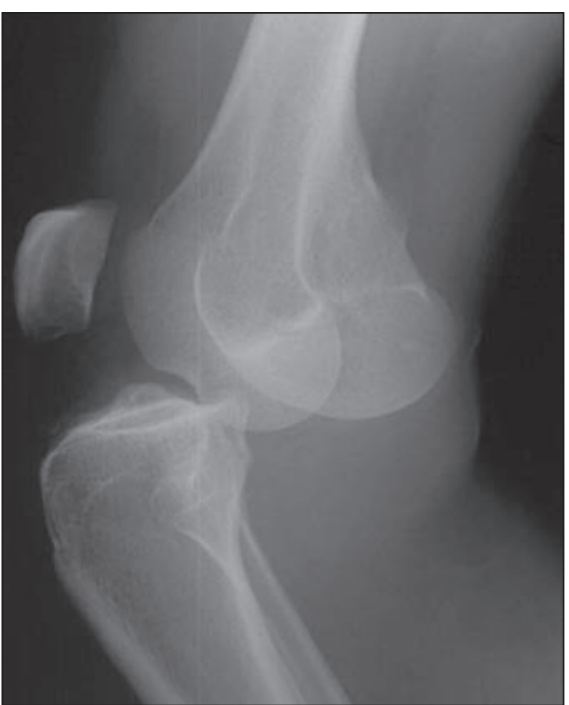

A

nonprofessionals; there were no competitionor jump-related downhill skiing accidents. All cases were considered to be caused by low-energy trauma.

The annual incidence of knee dislocation was calculated by comparing the patients with knee dislocation identified in our trauma center with the general population. This is a reasonable estimate because our hospital is the only one in an $8,929 \mathrm{~km}^{2}$ area that takes care of major trauma patients, such as those with knee dislocation. In case a patient with a knee dislocation is initially taken to some other hospital, the patient is eventually transferred to our hospital for final treatment. The entire population is entitled to a general health care system, and there are no private hospitals taking care of trauma patients in this area. No patients younger than 16 years are treated at our hospital. However, a traumatic knee dislocation is rare in the pediatric population. Therefore, the lack of identified pediatric dislocations most likely does not have a significant effect on its incidence. Therefore, we think that the defined approximation of the incidence of dislocated knees is useful and informative.

\section{MRI Findings and Injury Patterns}

Prior reports have shown that almost every patient with a knee dislocation has ruptured both cruciate ligaments [1, 6, 8-10]. Some studies have reported cases in which one of the cruciate ligaments has been intact $[1,18$, 19]. Usually the intact cruciate ligament is the PCL [18], but rare cases of knee dislocation without ACL disruption have been reported [19]. The PCL prevents the posterior

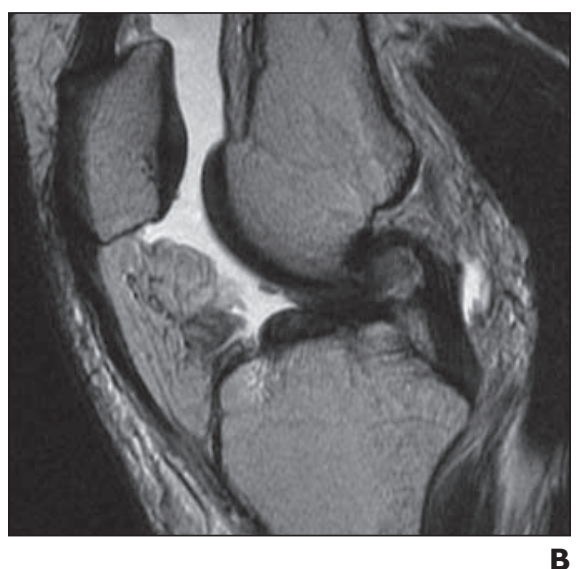

displacement of the knee, and the cases in which the PCL has been spared have been anterior or anteromedial knee dislocations $[9,18]$. Our findings are in agreement with those of previous studies. In our series, every patient had a torn ACL, and one patient with anterior dislocation of the knee had an intact PCL (Fig. 3).

In previous studies, the incidence of meniscal tears has ranged between $13 \%$ and $53 \%$ in medial menisci and between $7 \%$ and $71 \%$ in lateral menisci $[6,8,9]$. In our study, $18 \%$ of patients had a medial meniscal tear and $36 \%$ had a lateral meniscal tear. Chondral injuries have been reported to occur in $57 \%$ of patients [9]. In our series, $55 \%$ of patients had chondral injury.

In previous studies, $20-25 \%$ of patients with knee dislocation had severe injury to the peroneal nerve $[1,20]$. In our series, three of 24 patients (13\%) had complete peroneal palsy. Severe MRI findings of peroneal injury, such as an encasing hematoma or partial disruption of fibers, also display some degree of symptomatic peroneal nerve injury [9]. In our series, we found a good correlation between patients who had normal peroneal nerve findings on MRI and patients with no clinical signs or symptoms of peroneal nerve injury. If a hematoma surrounded an intact peroneal nerve, two of seven patients had symptoms of partial nerve injury; both of these patients had a complete neurologic recovery during follow-up. Of the three patients with complete irreversible peroneal paresis, only one underwent MRI before surgery. MR images in that patient showed severe hematoma along the course of the com-
Fig. 3-35-year-old woman with dislocated knee. A. Anterior dislocation of knee is seen on conventional radiograph.

B, Usually patients with complete dislocation of knee have ruptured both cruciate ligaments, but in this patient MR images show intact posterior cruciate ligament (PCL). At surgery, PCL was found to be intact.

mon peroneal nerve, and the nerve was not identified. However, we cannot make reliable conclusions based on a single case (Fig. 4).

When we compared injuries between groups $\mathrm{A}$ and $\mathrm{B}$, the only statistically significant difference was that in group B there were five complete ruptures of the popliteal tendon, and in group A every patient had an intact popliteal tendon. It has been suggested that the varus type of injury that results in a popliteal tendon rupture also increases the possibility of peroneal nerve injury [8]. Our findings are in agreement with that hypothesis. All three patients who had irreversible peroneal nerve injury were in group $\mathrm{B}$.

The low incidence $(13 \%)$ of major popliteal artery injury in our series may be explained by the high proportion of low-energy trauma. Note that, because arteriography was performed only in the setting of a clinical indication, the true incidence of popliteal artery injury has not been evaluated because a population of intimal injuries that did not progress to clinical signs would not be detected in this study.

\section{Image Quality}

A dedicated knee coil was used in each patient, and the subjective quality of all MR images was assessed as diagnostic. We did not find a significant difference in the number of false-positive or false-negative results between groups $\mathrm{A}$ and $\mathrm{B}$.

\section{Conclusion}

Eleven of 24 patients (46\%) with knee dislocation were overweight and sustained lowenergy trauma; a simple fall was a common cause. These patients had no injuries to the popliteal tendon or the peroneal nerve. Only $13 \%$ of 24 patients had major popliteal artery injury. Obesity did not interfere with the performance or subjective image quality of knee MRI examinations. As the prevalence of obesity increases, it may alter the injury patterns 


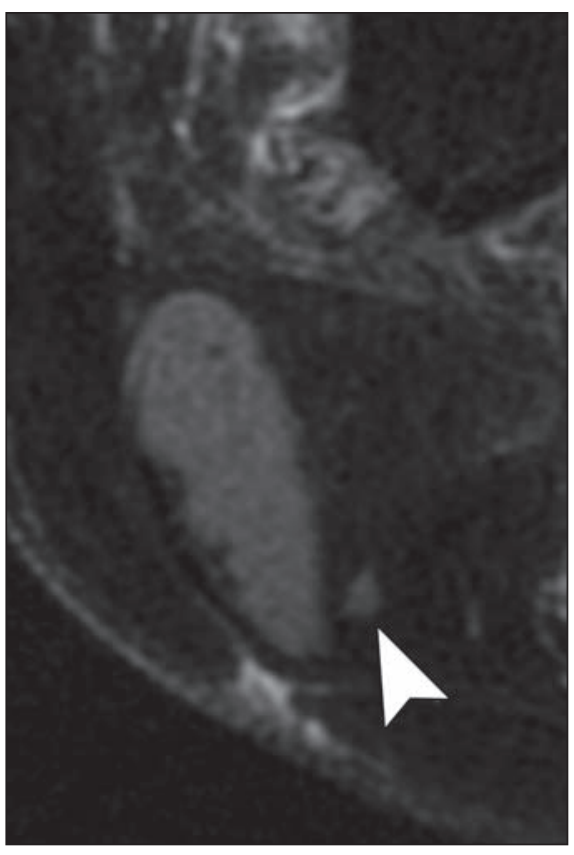

A
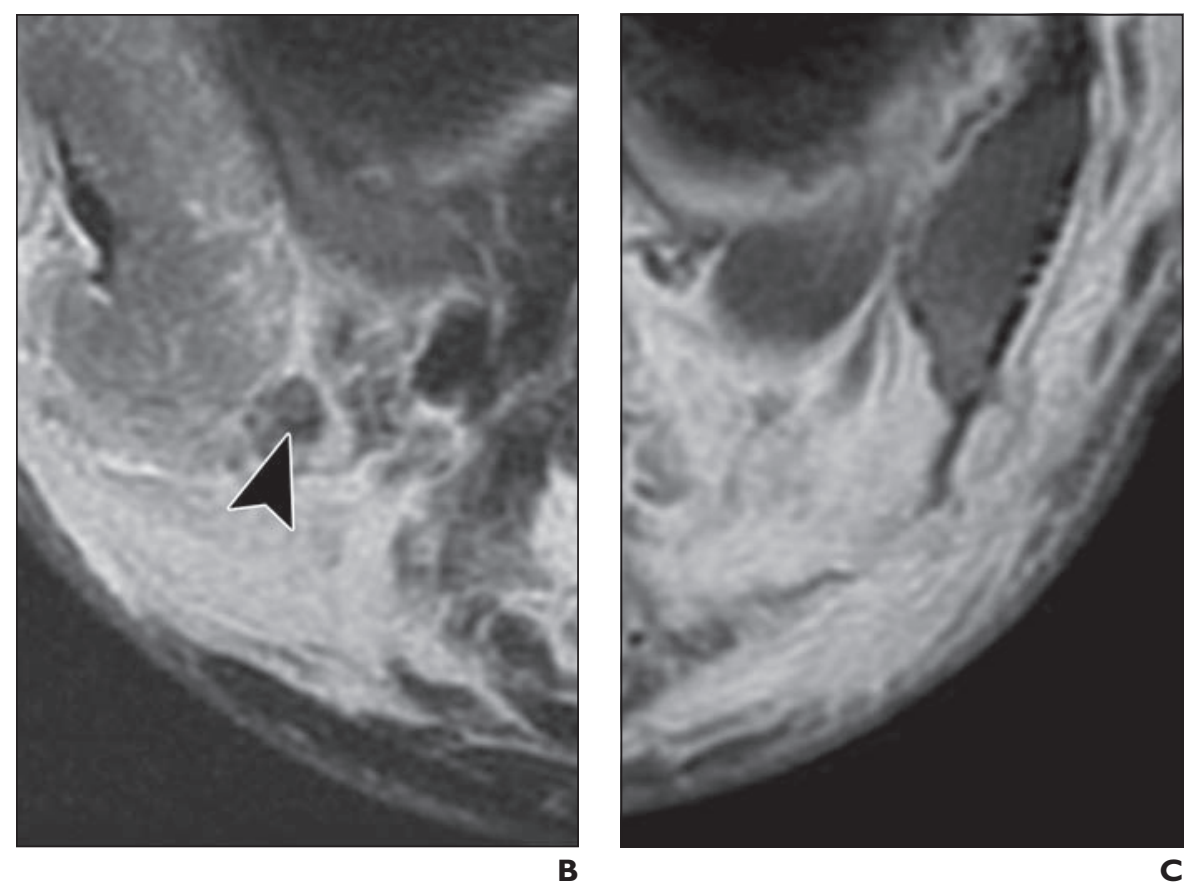

Fig. 4-MR images of three patients with knee injuries

A, In 39-year-old woman, common peroneal nerve (arrowhead) is intact and there is no surrounding hematoma. This patient had no signs or symptoms of nerve injury. B, In 29-year-old man, hematoma is seen around common peroneal nerve (arrowhead), but nerve seems to be intact. On initial physical examination, weak dorsiflexion of ankle was noted, but at follow-up no signs of nerve injury were seen.

C, In 16-year-old boy, MR image shows severe hematoma along course of common peroneal nerve, which cannot be identified. Patient had complete peroneal paresis and had not recovered at 1-year follow-up.

seen in emergency departments. The radiologist should be aware that, even after a simple fall, these patients may have a knee dislocation.

\section{Acknowledgment}

We thank Sharon J. Kuong for her linguistic expertise.

\section{References}

1. Robertson A, Nutton RW, Keating JF. Dislocation of the knee. J Bone Joint Surg Br 2006; 88:706-711

2. Armstrong PJ, Franklin DP. Management of arterial and venous injuries in the dislocated knee. Sports Med Arthrosc Rev 2001; 9:219-226

3. Reckling FW, Peltier LF. Acute knee dislocations and their complications: 1969. Clin Orthop Relat Res 2004; 422:135-141

4. Hollis JD, Daley BJ. 10-year review of knee dislocations: is arteriography always necessary? $J$ Trauma 2005; 59:672-675; discussion 675-676

5. Archbold HA, Sloan S, Nicholas R. A tibial plateau fracture in a knee dislocation: a subtle sign of major ligamentous disruption. Injury 2004; 35:945-947

6. Twaddle BC, Hunter JC, Chapman JR, Simonian PT, Escobedo EM. MRI in acute knee dislocation.: a prospective study of clinical, MRI, and surgical findings. J Bone Joint Surg Br 1996; 78: 573-579
7. Perron AD, Brady WJ, Sing RF. Orthopedic pitfalls in the ED: vascular injury associated with knee dislocation. Am J Emerg Med 2001; 19:583-588

8. Yu JS, Goodwin D, Salonen D, et al. Complete dislocation of the knee: spectrum of associated soft-tissue injuries depicted by MR imaging. AJR $1995 ; 164: 135-139$

9. Potter HG, Weinstein M, Allen AA, Wickiewicz TL, Helfet DL. Magnetic resonance imaging of the multiple-ligament injured knee. J Orthop Trauma 2002; 16:330-339

10. Twaddle BC, Bidwell TA, Chapman JR. Knee dislocations: where are the lesions? A prospective evaluation of surgical findings in 63 cases. $J$ Orthop Trauma 2003; 17:198-202

11. Shetty RR, Mostofi SB, Housden PL. Knee dislocation of a morbidly obese patient: a case report. $J$ Orthop Surg (Hong Kong) 2005; 13:76-78

12. Pace A, Fergusson C. Spontaneous non-traumatic dislocation of the knee. Acta Orthop Belg 2004; 70:498-501

13. Ogden CL, Carroll MD, Curtin LR, McDowell MA, Tabak CJ, Flegal KM. Prevalence of overweight and obesity in the United States, 19992004. JAMA 2006; 295:1549-1555

14. Stamatakis E, Primatesta P, Chinn S, Rona R, Falascheti E. Overweight and obesity trends from
1974 to 2003 in English children: what is the role of socioeconomic factors? Arch Dis Child 2005; 90:999-1004

15. Lissau I, Overpeck MD, Ruan WJ, et al. Body mass index and overweight in adolescents in 13 European countries, Israel, and the United States. Arch Pediatr Adolesc Med 2004; 158:27-33

16. Uppot RN, Sahani DV, Hahn PF, Gervais D, Mueller PR. Impact of obesity on medical imaging and image-guided intervention. AJR 2007; 188: 433-440

17. Brittberg M, Winalski CS. Evaluation of cartilage injuries and repair. J Bone Joint Surg Am 2003; 85-A[suppl 2]:58-69

18. Cooper DE, Speer KP, Wickiewicz TL, Warren RF. Complete knee dislocation without posterior cruciate ligament disruption: a report of four cases and review of the literature. Clin Orthop Relat Res 1992; 284:228-233

19. Flowers A, Copley LA. High-energy knee dislocation without anterior cruciate ligament disruption in a skeletally immature adolescent. Arthroscopy 2003; 19:782-786

20. Niall DM, Nutton RW, Keating JF. Palsy of the common peroneal nerve after traumatic dislocation of the knee. J Bone Joint Surg Br 2005; 87 ; 664-667 